\title{
Risiko im Anflug? Die Regulierung ziviler Drohnen
}

Silvio Hänsenberger / Isabelle Wildhaber *

Gesetzliche Bestimmungen beeinflussen die Entwicklung von neuen Technologien massgeblich. Im internationalen Wettbewerb ist bei der Regulierung von zivilen Drohnen zwischen wirtschaftlichen Interessen und Schutzbedürfnissen von Bevölkerung und Umwelt abzuwägen.

I. Einleitung. .83

II. Regulierungsbestrebungen in den USA und der EU.....................................83

1. Liberalisierung ziviler Drohnen in den USA.................................................84

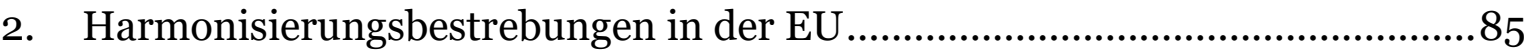

III. Regulierung ziviler Drohnen in der Schweiz...............................................86

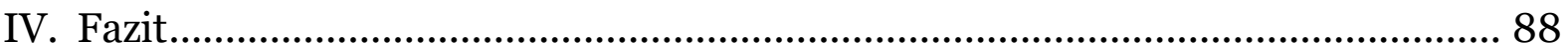

Zitiervorschlag: Silvio Hänsenberger / Isabelle Wildhaber, Risiko im Anflug? Die Regulierung ziviler Drohnen, in: sui-generis 2016, S. 82

URL: $\quad$ sui-generis.ch/26

DOI: $\quad$ https://doi.org/10.21257/sg.26

* Silvio Hänsenberger, M.A. HSG in law and economics, arbeitet am Forschungsinstitut für Arbeit und Arbeitswelten (FAA-HSG) und schreibt eine Dissertation zum Thema «Zivile autonome Drohnen».

Isabelle Wildhaber ist ordentliche Professorin für Privat- und Wirtschaftsrecht an der Universität St.Gallen.

Dieses Werk ist lizenziert unter einer Creative Commons Namensnennung - Weitergabe unter gleichen Bedingungen 4.0 International Lizenz. 


\section{Einleitung}

1 Drohnen ${ }^{1}$ sind auf dem Vormarsch. Das Bundesamt für Zivilluftfahrt (BAZL) schätzt, dass in der Schweiz über 20‘00o zivil, d.h. nicht militärisch, genutzte Drohnen in Betrieb sind. ${ }^{2}$ Die Mehrheit davon verfügt über ein Startgewicht von 0,5 kg bis 8 kg. 3 Zentrales Element einer Drohne ist die Steuereinheit. 4 Diese kontrolliert mit Hilfe eines Algorithmus den Flug.5 Je nach Entwicklungsstufe übernimmt sie die Kontrolle bei der Stabilisierung oder auch bei ganzen Flugmanövern. ${ }^{6}$ Ohne direkte Steuerung durch einen Piloten sind dadurch beispielsweise Schweben an Ort, Start und Landung, selbständiges Fliegen, das Ausweichen von Hindernissen, oder das Aufgreifen von Paketen möglich.7 Dadurch eröffnet sich ein breites kommerzielles Anwendungsfeld im Bereich von Logistik,

1 Für Drohnen werden auch die Bezeichnungen «unbemannte Luftfahrzeuge» (englisch «unmanned aerial vehicles», UAVs), (unbemannte Luftfahrzeugsysteme» (englisch «unmanned aircraft systems», UASs) oder als durch Piloten ferngesteuerte Luftfahrzeugsysteme (englisch «remotely piloted aircraft systems», RPAS) verwendet. Eine Drohne kann definiert werden als Fluggerät, welches ohne Pilot an Bord ferngesteuert wird oder autonom navigiert (ausführlich zur Bezeichnung (Drohne〉 siehe: Martin Steiger, Regulierung von Drohnen im zivilen Behördeneinsatz in der Schweiz, Sicherheit \& Recht 3/2014, S. 169 ff., S. 171 f.).

2 Bundesamt für Zivilluftfahrt, Zivile Drohnen in der Schweiz, 7.2.2016, S. 5.

3 BAZL (Fn. 2), S. 35.

4 Charles Pippin, Integrated Hardware/Software Architectures to Enable UAVs for Autonomous Flight. in: Kimon P. Valavanis/George J. Vachtsevanos (Hrsg.), Handbook of Unmanned Aerial Vehicles, Volume 3, Dordrecht 2015, S. 1725 ff., S. 1727.

5 Yasmina Bestaoui Sebbane, Smart autonomous aircraft, Flight control and planning for UAV, Boca Raton/London/New York, 2016, S. $201 \mathrm{f}$.

6 Vgl. Pippin (Fn. 4), S. 1727.

7 Henry H. Perrit/Eliot O. Sprague, Drones, Vanderbilt Journal of Entertainment and Technology Law 3/2015, S. $688 \mathrm{f}$.
Überwachung und Inspektionen, Landwirtschaft, Medien und Unterhaltung, humanitärer Hilfe, Schutz und Rettung, sowie Bau und Ingenieurwesen. ${ }^{8}$

2 Der zivile Einsatz von Drohnen verspricht grosses wirtschaftliches Potential. 9 Die Europäische Kommission erwartet im Bereich der Drohnentechnologie in den nächsten 10 Jahren einen Umsatz von rund EUR 15 Milliarden pro Jahr sowie bis zu 150`000 neue Arbeitsplätze. ${ }^{10}$ In den USA rechnet eine Branchenstudie mit einem Umsatzwachstum von USD 82,1 Milliarden und geht zudem von rund 104'000 neuen Arbeitsplätzen bis zum Jahr 2025 aus. ${ }^{11}$

\section{Regulierungsbestrebungen in den USA und der EU}

3 Zum heutigen Zeitpunkt existiert auf supranationaler Ebene kein umfassendes Regelwerk für die zivile unbemannte Luftfahrt. ${ }^{12}$ Die International Civil Aviation Organization (ICAO) hat eine Arbeitsgruppe zu diesem Thema eingesetzt, welche ein Handbuch für durch Piloten ferngesteuerte Luftfahrtsysteme erarbei-

$8 \longdiv { \text { Siddhartha Arora, Swiss Commercial Drone Indus- } }$ try, A possibility with potential?, Zürich/St.Gallen 2016, S. 29 ff.

$9 \quad$ BAZL (Fn. 2), S. 11.

10 Europäische Kommission, Mitteilung der Kommission an das Europäische Parlament und den Rat «Ein neues Zeitalter der Luftfahrt - Öffnung des Luftverkehrsmarktes für eine sichere und nachhaltige zivile Nutzung pilotenferngesteuerter Luftfahrtsysteme», 8.4.2014, S. 3 f.

11 Association for Unmanned Vehicle Systems International, The Economic Impact of Unmanned Aircraft Systems Integration in the United States, März 2013, S. 2.

12 Es gibt lediglich vereinzelte Bestimmungen wie z.B. Art. 8 Übereinkommen über die internationale Zivilluftfahrt, abgeschlossen in Chicago am 7.12.1944, in Kraft getreten für die Schweiz am 4.4.1947 (Stand am 25.2.2016), SR 0.748.0. 
tet hat. ${ }^{13}$ Darin wird im Kern die Gleichstellung der unbemannten mit der bemannten Luftfahrt gefordert. ${ }^{14}$ Allerdings enthält dieses Handbuch lediglich Empfehlungen. 15

\section{Liberalisierung ziviler Drohnen in den USA}

4 Das US-amerikanische Recht unterscheidet bei den zivilen Drohnen zwischen Freizeitdrohnen und kommerziell genutzten Drohnen. Für Letztere galten bislang grundsätzlich die Zulassungsbestimmungen der bemannten Luftfahrt. ${ }^{16}$ Ausnahmen von diesen strikten Vorgaben waren seit dem Jahr 2012 zwar möglich. ${ }^{17}$ Allerdings musste nach wie vor jede kommerzielle Nutzung durch die USamerikanische Luftfahrtbehörde (Federal Aviation Administration, FAA) bewilligt werden. ${ }^{18}$ Nach den bisher in den USA geltenden Gesetzen wäre es kaum möglich gewesen, die hohen wirtschaftlichen Erwartungen (oben I.) zu erfüllen. Per 29.8.2016 traten nun gelockerte Bestimmungen in Kraft. ${ }^{19} \mathrm{Neu}$ sind kommerzielle Flüge mit Drohnen bis 55 Pfund (ca.

$13 \overline{\text { International Civil Aviation Organization, Manual }}$ on Remotely Piloted Aircraft Systems (RPAS), 2015.

14 Z.B. International Civil Aviation Organization (Fn. 13), Ziff. 1.3.1, 1.5.1.

15 International Civil Aviation Organization (Fn. 13), Ziff. 1.2.10.

16 Konstantinos Dalamagkidis, Aviation Regulation, in: Kimon P. Valavanis/George J. Vachtsevanos (Hrsg.), Handbook of Unmanned Aerial Vehicles, Volume 4, Dordrecht 2015, S. 2117 ff., S. 2122 f.

17 Section 333 of the FAA Modernization and Reform Act of 2012 (FMRA), Public Law 112-95, 126 STAT. 11.

18 Bart Elias, Unmanned Aircraft Operations in Domestic Airspace: U.S. Policy Perspectives and the Regulatory Landscape, 27.1.2016, S. 8 ff.

19 Für den vollständigen Erlass siehe: Rule by the Federal Aviation Administration vom 28.6.2016, Operation and Certification of Small Unmanned Aircraft Systems, 81 FR 4203.
$25 \mathrm{~kg}$ ) ohne spezielle Bewilligung möglich. Eine Voraussetzung dafür ist, dass der Pilot einen «Drohnen-Pilotenschein» erworben hat. Die neuen US-Regelungen beinhalten aber noch weitere Einschränkungen: Zum Beispiel die Pflicht des Piloten, direkten Sichtkontakt zur Drohne $\mathrm{zu}$ halten (visual-line-of-sight, VLOS), ein Nachtflugverbot, ein Verbot über (nicht am Drohnenflug beteiligte) Menschen zu fliegen, sowie die Pflicht, eine maximale Flughöhe von 400 Fuss (ca. $122 \mathrm{~m}$ ) einzuhalten. ${ }^{20}$

5 In der Drohnenindustrie und bei möglichen kommerziellen Anwendern in den USA wird die Gesetzesanpassung als erster Schritt in die richtige Richtung begrüsst. ${ }^{21}$ Allerdings wird kritisiert, dass zum Beispiel aufgrund des VLOSErfordernisses kommerzielle Anwendungen mit grossem wirtschaftlichem Potential, wie Paketlieferdienste, nicht möglich seien. ${ }^{22}$ Daneben seien Flüge in urbanen Gebieten wegen des Überflugverbots von Menschen nahezu ausgeschlossen. ${ }^{23}$ Kritiker der FAA-Regulierung befürchten zudem, dass die USA mit dieser strikten Drohnenregulierung in wirtschaftlicher Hinsicht gegenüber Europa ins Hintertreffen geraten könnte. ${ }^{24}$

$2 0 \longdiv { \text { Vgl. dazu die Zusammenfassung: Federal Aviation } }$ Administration, FAA News, Summary of Small Unmanned Aircraft Rule (Part 107), 21.6.2016.

21 Ben Popper, New FAA rules mean US companies can fly drones without a pilot's license, The Verge 21.6.2016.

22 Cecilia Kang, F.A.A. Issues Commercial Drone Rules, The New York Times 21.6.2016.

23 Graham Warwick, FAA Urged To Act Fast On Final Small-UAS Rule, AVIATIONWEEK 15.2.2015.

24 Perlette M. Jura/William J. Peters/David A. Wolber, One Small Step or One Giant Leap? FAA Releases Final Rules on Commercial Drone Use in the United States, Gibson Dunn Publications 27.6.2016. 


\section{Harmonisierungsbestrebungen in der EU}

6 In der Europäischen Union fallen unbemannte Luftfahrzeugsysteme bis $150 \mathrm{~kg}$ in den Zuständigkeitsbereich der Mitgliedstaaten. ${ }^{25}$ Deshalb existieren heute in diesem Bereich grosse gesetzliche Unterschiede zwischen den einzelnen Mitgliedsländern. ${ }^{26}$ Allerdings sind Bestrebungen zu einer einheitlichen Regelung für den gesamten EU-Binnenmarkt im Gang. ${ }^{27}$ Diesbezüglich arbeitet die Expertengruppe JARUS (Joint Authorities for Rulemaking on Unmanned Systems) innerhalb der Europäischen Agentur für Flugsicherheit (EASA) an harmonisierten Bestimmungen für zivile Drohnen. ${ }^{28}$ Über den Stand dieser Arbeiten wurde im Juni 2016 in einem nicht verbindlichen Papier (Non-Paper: Roadmap for drone operations in the European Union) ${ }^{29}$ berichtet.30 Demnach sollen zum

$25 \overline{\text { Verordnung (EG) Nr. 216/2008 des Europäischen }}$ Parlaments und des Rates vom 20.2.2008 zur Festlegung gemeinsamer Vorschriften für die Zivilluftfahrt und zur Errichtung einer Europäischen Agentur für Flugsicherheit, zur Aufhebung der Richtlinie 91/670/EWG des Rates, der Verordnung (EG) Nr. 1592/2002 und der Richtlinie 2004/36/EG, Anhang 2 Buchstabe i.

26 European Parliament, Committee on Transport and Tourism, Report on safe use of remotely piloted aircraft systems (RPAS), commonly known as unmanned aerial vehicles (UAVs), in the field of civil aviation, 19.6.2015, S. 3 f.

27 European Parliament, Committee on Transport and Tourism (Fn. 26), S. 7.

28 Europäische Kommission (Fn. 10), S. 6 f.; BAZL (Fn. 2), S. 19 f.; Michael Funke, EUKommission: Regelungen für zivilen Drohneneinsatz, Computer und Recht 5/2014, S. 54 ff.; Arora (Fn. 8), 62 f.; Steiger (Fn. 1), S. 176.

29 European Aviation Safety Agency, Non-Paper: Roadmap for drone operations in the European Union (EU), The roll-out of the EU operation centric approach, 20.6.2016.

30 Das Non-Paper (Fn. 29) wurde am 20.6.2016 am RPAS Stakeholders Workshop am Hauptsitz der European Aviation Safety Agency in Köln
Beispiel Kleindrohnen (bis $25 \mathrm{~kg}$ ) ${ }^{31}$ getrennt von der bemannten Luftfahrt fliegen, d.h. bis maximal $150 \mathrm{~m}$ Höhe ${ }^{22}$ und nicht im Umkreis von Flugplätzen und Flughäfen33. Flüge ausserhalb der Sichtweite des Piloten sollen möglich sein (beyond-line-of-sight, BLOS).34 Dafür wird allerdings ein «sense and avoid»System vorausgesetzt.35 Dazu müssen Drohnen mit Sensoren ausgerüstet sein, welche andere Luftverkehrsteilnehmer erkennen können. Ein Steuercomputer an Bord der Drohne wertet die Sensordaten aus und leitet bei Kollisionsgefahr ein Ausweichmanöver ein. ${ }^{36}$ Zurzeit existieren allerdings noch keine marktreifen «sense and avoid»-Systeme. 37 Der definitive Entwurf für ein Betriebskonzept von unbemannten Luftfahrzeugen in der EU wird in den nächsten Monaten erwartet und soll ab dem Jahr 2018 in Kraft treten. 38
(Deutschland) präsentiert, siehe UAS Vision, EASA's «Non-Paper» Roadmap for EU Drone Operations, ohne Datum.

31 European Aviation Safety Agency (Fn. 29), S. 20.

32 European Aviation Safety Agency (Fn. 29), S. 1.

33 European Aviation Safety Agency (Fn. 29), S. 6.

34 European Aviation Safety Agency (Fn. 29), S. 28 f.

35 European Aviation Safety Agency (Fn. 29), S. 12, Fn. 28.

36 Ausführlich dazu z.B. Jörg Meyer/Matthias Göttken/Christoph Vernaleken/Simon Schärer, Automatic Traffic Alert and Collison Avoidance System (TCAS) Onboard UAS, in: Kimon P. Valavanis/George J. Vachtsevanos, Handbook of Unmanned Aerial Vehicles, Volume 4, Dordrecht 2015, S. $1857 \mathrm{ff}$.

$37 \mathrm{Zu}$ Entwicklungsbemühungen im Bereich «sense and avoid»vgl. Tom Abate, Will drones get their own air traffic control?, Futurity 11.12.2015; Safran Group, Sagem demonstrates Patroller drone's ability to operate in civilian airspace, 12.12.2015.

38 European Aviation Safety Agency (Fn. 29), S. 18. 


\section{Regulierung ziviler Drohnen in der Schweiz}

7 Im Rahmen des Luftverkehrsabkommens zwischen der Schweiz und der EU39 sind europäische Erlasse im Bereich des zivilen Luftrechts auch für die Schweiz von Bedeutung.40 Deshalb sind Vertreter des BAZL aktiv beim Projekt JARUS beteiligt. 41

8 In der Schweiz können Drohnen bis $30 \mathrm{~kg}$ unabhängig ihres Einsatzzweckes grundsätzlich ohne Bewilligung betrieben werden (Art. 14 Abs. 1 VLK42 e contrario). Zwar gelten auch hier Einschränkungen für Drohnen zwischen $0,5 \mathrm{~kg}$ und $30 \mathrm{~kg}$ wie die VLOS-Pflicht (Art. 17 Abs. 1 VLK), das Verbot von Flügen über Menschenansammlungen (Art. 17 Abs. 2 lit. c VLK) und eine Versicherungspflicht (Art. 20 Abs. 1 VLK). 43 Von

39 Abkommen zwischen der Schweizerischen Eidgenossenschaft und der Europäischen Gemeinschaft über den Luftverkehr, abgeschlossen am 21. 6.1999, in Kraft getreten am 1.6.2002 (Stand am 15.5.2016), SR 0.748.127.192.68 (zit. Luftverkehrsabkommen Schweiz-EU).

40 Das Luftverkehrsabkommen Schweiz-EU (Fn. 39) war Teil der Bilateralen Abkommen I (1999). Eine automatische Rechtsübernahme ist darin nicht vorgesehen. Art. 21 des Luftverkehrsabkommen Schweiz-EU (Fn. 39) bezeichnet einen Gemischten Ausschuss, dem der Informationsaustausch über Rechtsänderungsintentionen einer Vertragspartei obliegt. Sind Anpassungen im Schweizer Recht an europäische Erlasse erforderlich, sind diese über das Schweizer Gesetzgebungsverfahren in Kraft zu setzen (vgl. zur Rechtsübernahme im Allgemeinen: Michael Meyer, Die Übernahme europäischen Rechts durch die Schweiz, in: Astrid Epiney/Markus Kern/Lena Hehemann, Schweizerisches Jahrbuch für Europarecht 2014/2015, Zürich 2015, S. 347 ff.).

41 BAZL (Fn. 2), S. $19 \mathrm{f}$

42 Verordnung des UVEK über Luftfahrzeuge besonderer Kategorien (VLK) vom 24.11.1994 (Stand am 15.7.2015), SR 748.941.

43 Siehe ausführlich dazu Martin Steiger, Drohnen: Schweiz verschärft Regeln per 1. August 2014,
Einschränkungen wie der VLOS-Pflicht erteilt das BAZL Ausnahmenbewilligungen (Art. 18 VLK). Neben weiteren Auflagen muss dafür eine umfassende Risikoanalyse einen genügenden Schutz von Bevölkerung und Umwelt feststellen (Art. 18 Abs. 2 VLK). 44 Somit sind - wie in den USA (oben II.A) - beispielsweise Drohnen-Paketlieferdienste, wie sie durch Matternet, Swiss Cargo und die Schweizerische Post im letzten Jahr getestet wurden, 45 nur mit einer Ausnahmebewilligung möglich.

9 Neben luftrechtlichen Regulierungen schränken weitere Bestimmungen die Nutzung von Drohnen ein. Werden zum Beispiel mittels Drohnenkameras Personen aufgenommen, können diese Bilder als Personendaten im Sinne von Art. 3 lit. a DSG46 gelten. In diesem Fall kommen die Einschränkungen des Datenschutzgesetzes zur Anwendung.47 Das Eindringen mit einer Drohne in den Privatbereich einer Person kann zudem eine Verletzung des Persönlichkeitsrechts nach Art. 28 ZGB 48 darstellen. 49 Ebenso

Steiger Legal 10.7.2014.

44 BAZL (Fn. 2), S. $14 \mathrm{ff}$.

45 Michael Schoenenberger, Drohnen sollen Pakete zustellen: Die Post geht in die Luft, NZZ Online 7.7.2015

46 Bundesgesetz über den Datenschutz (DSG) vom 19.6.1992 (Stand am 1.1.2014), SR 235.1.

47 Weiterführend Rolf H. Weber/Dominic Oertly, Datenschutzrechtliche Problemfelder von zivilen Drohneneinsätzen, Jusletter 26.10.2015, S. 1 ff.; Berthold H. Haustein, Herausforderungen des Datenschutzrechtes vor dem Hintergrund aktueller Entwicklungen in der Robotik, in: Eric Hilgendorf/Jan-Philipp Günther (Hrsg.), Robotik und Gesetzgebung, Beiträge der Tagung vom 7. bis 9. Mai 2012 in Bielefeld, Baden-Baden 2013, S. 93 ff.

48 Schweizerisches Zivilgesetzbuch (ZGB) vom 10.12.1907 (Stand am 1.4.2016), SR 210.

49 Weiterführend zum Recht auf Achtung der Intimund Privatsphäre (Art. 28 ZGB) siehe: Andreas Meili, Basler Kommentar, Zivilgesetzbuch I, Art. 1- 
können beim Drohnenüberflug privater Grundstücke sachenrechtliche Abwehransprüche bestehen.50 In diesem $\mathrm{Zu}-$ sammenhang wird auch das gerichtliche (Überflug-)Verbot als Abwehrinstrument ins Feld geführt, ${ }^{51}$ auch wenn die Praktikabilität dieses Lösungsansatzes anzuzweifeln ist. Gleichzeitig müssen Normen des Umweltschutzrechts wie die Lärmschutz-Verordnung 52 oder Regelungen zum Schutz von Wildtieren und Vögeln53 eingehalten werden. Schliesslich sind auch zoll- und steuerrechtliche Bestimmungen relevant, wenn zum Beispiel ausländische Drohnenbetreiber Dienstleistungen über Grenzen hinweg in der Schweiz anbieten. 54

Allerdings sind Drohnenpiloten oft schwierig zu eruieren, gerade wenn Modelle zum Einsatz kommen, die mittels Videoübertragung ausserhalb des Sicht-

456, Heinrich Honsell/Nedim Peter Vogt/Wolfgang Wieland (Hrsg.), 4. Aufl., Basel 2014, Art. 28 N 16 f., 23 ff. Konkret zur Beeinträchtigung durch Drohnen siehe: z.B. Krista Nadakavukaren Schefer, Ein völkerrechtlicher Schutz der kollektiven Privatsphäre? Der Schutz der Privatsphäre und die Anonymität im Zeitalter von Gewinn-orientierten Sammlungen von aggregierten Daten durch Drohnen, ZSR 2014 I, S. 259 ff.

50 Weiterführend Stephanie Hrubesch-Millauer/David Bruggisser, Sachenrechtliche Aspekte zum Einsatz von privaten Drohnen, Jusletter 11.8.2014; Daniel Kettiger, Das gerichtliche Verbot als Instrument zur Abwehr ziviler Drohnen, Jusletter 11.4.2016.

${ }^{51}$ Kettiger (Fn. 50), passim.

52 Lärmschutz-Verordnung (LSV) vom 15.12.1986 (Stand am 1.1.2016), SR 814.41.

53 So z.B. Verordnung über die Wasser- und Zugvogelreservate von internationaler und nationaler Bedeutung (WZVV) vom 21.1.1991 (Stand am 15.7.2015), SR 922.32, Bundesgesetz über die Jagd und den Schutz wildlebender Säugetiere und Vögel (JSG) vom 20.6.1986 (Stand am 1.1.2014), SR 922.0, oder Bundesgesetz über den Natur- und Heimatschutz (NHG) vom 1.7.1966 (Stand am 12.10.2014), SR 451.

54 BAZL (Fn. 2), S. 28 f. bereichs des Betreibers fliegen können (BLOS). Deshalb stehen den vorgenannten Bestimmungen zurzeit grosse Durchsetzungsprobleme gegenüber.

11 Aktuell wird beim BAZL nach Lösungen gesucht, wie illegal fliegende Drohnen ihrem Piloten zugeordnet werden können. Laut Urs Holdegger vom BAZL sollen Drohnen künftig mit einem Chip versehen werden, der geortet werden kann.55 Damit soll die Identifikation des Betreibers der Drohne möglich sein. In dieser Hinsicht scheint eine Registrierungspflicht für im Freien fliegende Drohnen in der Schweiz eine Frage der Zeit. Für Freizeitdrohnenpiloten in den USA ist eine Registrierungspflicht bereits Realität. Sie sind seit dem 19.2.2016 verpflichtet, ihre Drohne ab einem Gewicht von 0,55 Pfund (ca. 250 g) in einem nationalen Register einzutragen. 56

12 Ein solches Register kann ein erster Schritt für die Zulassung von (teil-) autonomen Flügen ausserhalb der Sichtweite des Piloten (BLOS) sein. Allerdings sind dazu weitere technische Entwicklungen notwendig. Es fehlen zum Beispiel marktreife «sense and avoid»Systeme (oben II.B). Für die Entwicklung solcher neuen Technologien sind Tests unter realen Bedingungen unabdingbar. Diese sind mittels Ausnahmebewilligungen in der Schweiz möglich, sofern dadurch Bevölkerung und Umwelt nicht gefährdet werden.

55 Ursina Haller, Registrierungspflicht: Drohnen sollen mit Chip fliegen, NZZ Online 14.7.2016.

56 Rule by the Federal Aviation Administration vom 16.12.2015, Registration and Marking Requirements for Small Unmanned Aircraft, 80 FR 78593. 


\section{Fazit}

13 Bei der Regulierung von neuen Technologien ist es wichtig, gut zwischen wirtschaftlichen Interessen sowie Schutzinteressen von Bevölkerung und Umwelt abzuwägen. In unseren Augen hält die Schweizer Lösung im Moment mit den technischen Möglichkeiten Schritt und lässt Spielraum für Ausnahmebewilligungen. Daran würde auch eine Registrierungspflicht nichts ändern. Durch die Rückverfolgbarkeit von fehlbaren Drohnenpiloten würde das Vertrauen der Bevölkerung in die Drohnentechnologie gestärkt, was sich letztlich positiv auf die Akzeptanz von Drohneneinsätzen auswirken würde. Der Bundesrat hat erst kürzlich beantragt, eine Motion abzulehnen, 57 welche eine weitergehende Regulierung von Drohnen verlangte. $5^{8} \mathrm{Im}$ Sinne eines starken Forschungs- und Technologie-Standortes Schweiz ist diese Haltung zu befürworten.

57 Stellungnahme und Antrag vom 6.7.2016 des Bundesrates zur Motion Leutenegger Oberholzer (16.3310): Drohnen. Bevölkerung vor Gefährdungen Schützen.

58 Motion Leutenegger Oberholzer (Fn. 57). 